\title{
Automatic Fruits Identification System Using Hybrid Technique
}

\author{
A. M. Aibinu, M. J. E. Salami, A. A. Shafie, N. Hazali and N. Termidzi \\ Department of Mechatronics Engineering, \\ International Islamic University Malaysia (IIUM) \\ P O Box 10, 50728,Gombak, Malaysia. \\ E-mail: maibinu@gmail.com
}

\begin{abstract}
In this work, a combination of artificial neural network (ANN), Fourier descriptors (FD) and spatial domain analysis (SDA) has been proposed for the development of an automatic fruits identification and sorting system. Fruits images are captured using digital camera inclined at different angles to the horizontal. Segmentation is used for the classification of the preprocessed images into two non-overlapping clusters from which shape boundary and signatures are estimated using FD and SDA technique. Furthermore, color information obtained from the extracted red-green-blue color components of the fruits images during ANN training process is used in accurately detecting the color of such a fruit. The two independent paths are then combined for fruits sorting and identification purposes. The performance of the developed hybrid system has been evaluated at three different angles of camera inclination from which an accuracy of $99.1 \%$ was obtained.
\end{abstract}

Keywords-Artificial Neural Network (ANN); Fruits Images; Fourier Descriptors; Shape.

\section{INTRODUCTION}

Fruits recognition and classification plays an important role in pre-harvesting and post-harvesting operations. Various techniques have been presented in the literature for fruits recognition and classification and these may be classified into: shape based; color based; dielectric based; conductivity based and more recently magnetic resonance imaging techniques. The shape based technique using hybrid Fourier descriptors (FD) and spatial domain analysis (SDA) is proposed in this paper.

Shape based image analysis has been an area of keen research interest during the last few decades especially in the field of medical image diagnosis, machine vision, content retrieval system, surveillance, target recognition, industrial inspection, scene analysis [1]-[11].

Shape representation simply involves characterization of a given shape using certain features [2]. The given shape is normally represented by a finite sequence of complex or real numbers, symbols, bits, features etc and these are called shape signatures [2]-[4]. Several shape boundary representative schemes do exist and these can be categorized into complex-valued signature (CVS) or real-valued signature (RVS).

The CVS representation can further be divided into two types of representation, namely centered based complex coordinates and non-centered based complex coordinates
[2]-[5], [8], [11]. The mathematically expression for the two types of CVS representation schemes are respectively given as:

$$
\begin{gathered}
z_{n}=x_{n}+i y_{n} \\
z_{n}=\left(x_{n}-x_{c}\right)+i\left(y_{n}-y_{c}\right)
\end{gathered}
$$

where $x_{c}, y_{c}$ refer to the coordinates of the center pixel and $x_{n}, y_{n}$ refer to the $n^{t h}$ boundary pixel of the shape. Similarly, RVS types include centroidal signature, farthest point signature, curvature signature, chord length etc [2][19]. Shape based techniques using centered-based CVS have been applied in the development of automatic fruit identification and sorting system in this work. The remaining part of this paper is organized as follows: review of some related work is covered in section II; the proposed technique is presented in section section III while results and conclusion is contained in section IV.

\section{LITERATURE REVIEW}

Ear identification using Generic Fourier Descriptor (GFD) has been proposed in [12]. This proposed technique has been found to be rotational and scale invariant. A three stage automatic teeth classification in bitewing images using Bayesian classification and FD of the contour of the molar and the premolar teeth was proposed in [20]. Contour of each of the individual teeth was obtained from the segmented images while the Bayesian and FD provided the initial classification. Spatial relationship between the images was then used for final classification. The maximum and minimum performance value obtained for pre-classification of molar or premolar (in mandible and maxilla) is $95.5 \%$ and $72 \%$ respectively. Ten low and high frequency components have been used to describe pedestrian and vehicle shapes in [17]. The application of the proposed algorithm on 500 pedestrians and 300 vehicles feature images shows that 20 coefficients is sufficient to represent all the necessary features from the acquired images.

An efficient two-stage shape based leaf image retrieval system has been proposed in [19]. Results obtained show that the proposed hybrid technique performs better than both the individual performance of the curvature scale space and the modified FD in identifying 1400 leaf images from 140 plants [19]. 


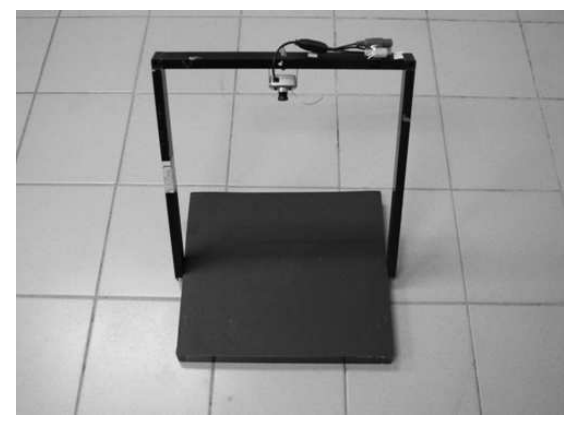

(a)

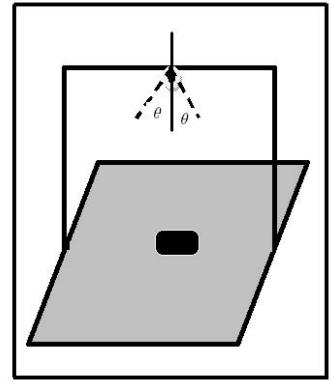

(b)

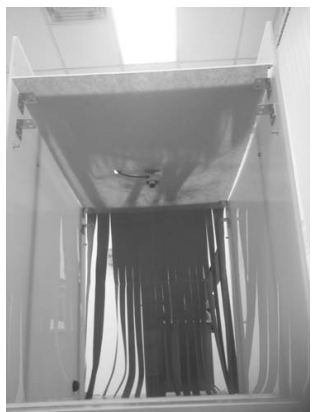

(c)
Figure 1. Stand alone fruits image acquisition system

Shape representation using autoregressive (AR) parametric modeling technique in digitized object images was proposed in [10]. Three different pattern recognition algorithms which assign object names to unlabeled sets of AR model parameters were tested and the obtained results show that the proposed techniques have the ability to accurately describe and recognize varieties of complex shapes using few parameters, thereby reducing the size of memory space needed for data storage by the classifier. Furthermore, the proposed technique has been shown to be invariant to shape size and orientation. In another work, shapes classification using neural network based autoregressive parametric modeling technique has been suggested in [21]. Complex-valued autoregressive model using complex-valued neural networks was proposed for complex boundary signature whereas realvalued neural networks has also been suggested for realvalued centroidal signatures.

\section{Methodology}

The methodology for the implementation of the proposed fruit sorting and identification system is presented herewith. Fruits images were captured using digital camera inclined at different angles to the horizontal. The stand alone fruit image acquisition system is shown in Fig. 1(a) and the schematic diagram of camera inclination is shown in Fig. 1(b). This stand alone is often affected by the lighting condition of the surrounding environment thus necessitating a control light intensity chamber in the fully hybrid system shown in Figure 1(c). The image filtering stage eliminates unwanted interference and noise associated with the acquired image. The fruit shape recognition uses the shape boundary in recognizing the fruit while color information obtained from the extracted red-green-blue color scheme during training process from artificial neural network (ANN) system is used in the recall phase in accurately detecting the color of such a fruit. The two results are then combined using logic gates system so as to sort and identify the fruit as subsequently described.

\section{1) Fruit Image (Data) Acquisition}

This stage is common to the two subsections and it involves the use of low cost digital camera in capturing the required fruits images. Fig. 1(a-c) show the fruit data acquisition system. Three different camera positions have been used for the data acquisition, namely: top view (with the camera inclined at $90^{\circ}$ to the horizontal); angular view (with the camera inclined at $60^{\circ}$ to the horizontal) and front view. The angle of inclination is as shown in Fig. 1b.

2) Fruit Color Recognition System

The fruit color recognition system consists of two sub-units, namely the training mode and the recall mode. During the training mode, the acquired fruit image is segmented into two different clusters based on color, these are the background section and the fruit section. The fruit section is then saved while the background image is eliminated. The mean value of the RGB component is extracted for different types of fruit and these are used for training a two-layer, three-inputs ANN. The same number of hidden nodes were used for both training and recall mode while the number of output nodes depend on the number of color segmentation required. A typical ANN consists of interconnection of simple processing elements called nodes with two types of layers namely, the hidden layer and the output layer. The first interface, though passive in nature is where data are fed to the network, which is called the input nodes. Another interface, where processed data are released out of the network is the output layer and between these two layers are the hidden layers. Each layer, except the input layer, consists of one or more processing unit [25].

In the recall mode, the color segmentation and extraction, as implemented under training mode are revisited. A radial basis ANN suitable for color classification and analysis is then applied in the determination of the color scheme. The result obtained from this mode is then compared with that in the database in 


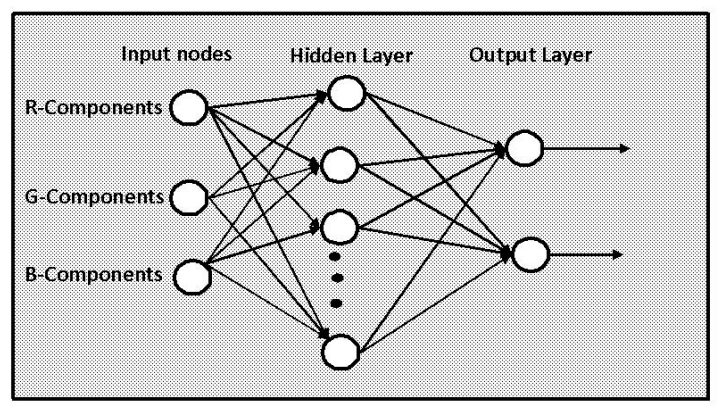

Figure 2. Artificial neural network used for fruits image color segmentation

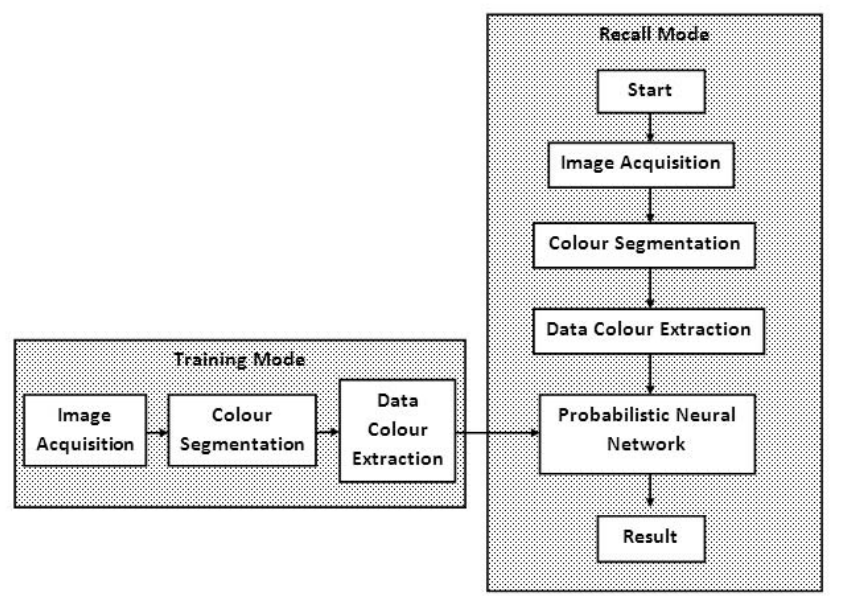

Figure 3. Fruit color recognition system

order to determine the appropriate color of the fruit. A typical ANN used is shown in Fig. 2.

\section{3) Fruit Shape Recognition System}

A four-stage approach for automatic fruits shape identification using FD and SDA techniques is proposed in this section and the detailed analysis of the steps involved are given subsequently.

a) Color Space Conversion:

The acquired RGB images were converted to Hue-Saturation and Intensity (HSI) using

$$
\begin{aligned}
H & = \begin{cases}\theta, & \text { for } B \leq G \\
360-\theta, & \text { for } B>G\end{cases} \\
S & =1-\left\{\frac{3}{R+G+B}\right\} \min (R, G, B) \\
I & =\frac{1}{3}(R+G+B) .
\end{aligned}
$$

where

$$
\theta=\cos ^{-1}\left\{\frac{[(R-G)+(R+B)]}{\left[(R-G)^{2}+(R-B)(G-B)\right]^{1 / 2}}\right\}
$$

and $\min (R, G, B)$ denotes the minimum of RGB components of the acquired input fruit image [1],
[3], [4]. The choice of HSI color space is based on the ease of its conversion and its similarity to gray level image color space conversion. Only the intensity (I) matrix is saved for further processing.

b) Fruit Image Segmentation and Boundary Extraction

Fruit image segmentation can be described as the partitioning of the intensity image obtained from the RGB components image into two nonoverlapping, connected, groups of pixels (regions) [1], [3], [4]. Here the fruit image segmentation involves the use of Otsu method and this is followed by morphological operation of erosion and dilation [23]. The last operation is the extraction of centered-based boundary coordinates.

c) Fruits Fourier Descriptor (FD)

This involves the application of Discrete Fourier Transform (DFT) on the obtained boundary signature. The FD representation decomposes a CBI into its frequency components by the application of DFT on the shape signature, that is

$$
A(k)=\sum_{n=0}^{N-1} z_{n} e^{-i 2 \pi \frac{k n}{N}}
$$

The complex valued coefficients $A(k), k=$ $0,1, \ldots, N-1$, are the FD of the shape [2]-[7], [12]-[19]. The lower FD coefficients have been shown to contain information about the general shape of the CBI while the high frequency FD coefficients contain information about small detail.

The inverse discrete Fourier transform (IDFT) of these complex coefficients restores back the original boundary coordinates,$z_{k}$, that is,

$$
z_{n}=\frac{1}{N} \sum_{k=0}^{N-1} A(k) e^{i 2 \pi \frac{k n}{N}}
$$

for $k=0,1,2 \ldots N-1$. Rotational invariant of the image has been obtained by dividing each of the FD coefficients by the DC term [12], [17], [18] while translational invariant descriptor was obtained by the use of centered CVS or centroidal distance signature.

The obtained FDs were normalized using

$$
\mid \frac{|A(1)|}{|A(0)|} ; \frac{|A(2)|}{|A(0)|} ; \frac{|A(3)|}{|A(0)|} ; \ldots ; \frac{|A(k)|}{|A(0)|}
$$

for $k=0,1,2, \ldots, P$ and $P$ is the optimal number of FD required for efficient representation of the fruit shape.

d) Fruits Spatial Domain Analysis

The fruits SDA involves the application of a set 
of geometric related criteria on the segmented boundary image. The criteria used are given as

\section{- Compactness Test (CT)}

Shape compactness test is given as

$$
C=\frac{4 \pi A}{P^{2}}
$$

where $\mathrm{A}$ is the area of the shape and $P$ is the shape perimeter [4].

\section{- Ratio Test (RT)}

The ratio test of a segmented fruit image is given as the ratio of segmented image minor axis to the segmented image major axis.

\section{- Eccentricity (EC)}

Eccentricity is defined as the ratio of the distance between the foci of the ellipse and its major axis length. It has a value between 0 and 1 , where an ellipse with eccentricity 0 is a circle and an ellipse with eccentricity 1 is a line segment [4], [22].

\section{REsults AND CONCLUSiON}

The main objective of this work is to recognisee and sort different fruits based on color and shape information. Three dissimilar fruits have been used for the evaluation of the proposed techniques, these are apple, banana and mango. The choice of these fruits is based on the dissimilarity in shape but similarity in color. The acquired fruits images using the proposed methodology discussed in section III were divided into two groups, namely training and test sets. The average values of the FD and SDA for each class of the three fruits were used as the class feature while the trained ANN has been used for color sorting and identification with the input being the mean of the various RGB components of the training images. In the test mode, images from the test set were assigned to the class with the minimal Euclidean distance between the image class features and the obtained test set image features.

Preliminary results in Fig. 4(a-r) show that better segmented images are obtainable using the top view and $60 \%$ angular camera inclination approach (Figure 1) than the use of front view (Fig. 4(m-r)). The real shape of the fruits cannot be captured using front view due to the problem of uneven illumination associated with the set up, hence the use of top view or $60 \%$ angular inclination is recommended for future fruit image data acquisition using the proposed technique.

Also evaluated in this work is the use of Ycbcr and MATLAB in-built gray level color space conversion techniques. The use of MATLAB in-built gray level technique shows almost similar performance with the use of HSI described in (3), hence the use of either HSI or MATLAB in-built grey level conversion can be adopted for related work.

Furthermore, it have also been observed that increasing the number of FDs greatly increase the accuracy and the computation time of the system, thus the highest classification accuracy of $99.1 \%$ was obtained in this work with 200 FDs. With lesser number of FDs, the accuracy varies between $96 \%$ and $98.5 \%$

The development of a more robust system so as to improve the system accuracy and to reduce the time of completion is under investigation. It is believed that the introduction of artificial intelligent based parametric modeling techniques and fruits color identification scheme to this present system would greatly increase the accuracy of the system.

\section{ACKNOWLEDGMENT}

This work is partially supported by Malaysian E-science grant $01-01-08-S F 0083$

\section{REFERENCES}

[1] A.M. Aibinu, M.I. Iqbal, A.A. Shafie, M.J.E. Salami and M. Nilsson "Vascular intersection detection in retina fundus images using a new hybrid approach", Computers in Biology and Medicine, Volume 40, Issue 1, 81-89, 2010.

[2] L. Costa and R. M Cesar Jr., "Shape Analysis and Classification", CRC Press, 2001.

[3] R. Gonzalez and R. Woods, "Digital Image Processing", Prentice-Hall Inc., 2002.

[4] A. M Aibinu, M. J. E Momoh, A. A. Shafie, M. Alli and I. A. Bamgbopa "Assessment of Mould Growth on Building material Using Spatial and Frequency Domain Analysis", Int. Journal of Computer Science and Network Security, pp 154-167, July 2009.

[5] H. Kauppinen, T. Seppanen, and M. Pietikainen, "An Experimental comparison of autoregressive and fourier-based descriptors in 2D shape classification", IEEE PAMI , vol. 17-2 pp. $201207,1995$.

[6] D. Zhang and G. Lu, " A Comparative Study of Fourier Descriptors for Shape Representation and Retrieval", in The 5th Asian Conf. on Computer Vision, pp. 1-6 , Jan. 2002.

[7] D. Zhang and G. Lu, " Review of Shape Representation and Description Techniques", in Elsevier, The Journal of the Pattern Recognition Society, Vol. 37, pp.1-19, 2004.

[8] C. Liu and N. Ahuja, " A model for Dynamic Shape and Its Applications”, IEEE Trans. , CVPR, Vol.2, Page(s):II-129 II-134 Vol. 2, 2004.

[9] R. L. Kashyap and R. Chellappa, "Stochastic Models for Closed Boundary Analysis, Representation and Reconstruction", in IEEE Trans. on Information Theory, Vol. IT-27, No. 5 pp. 627-637, Sep. 1981.

[10] S. R. Dubois and F. H. Glanz, "An Autoregressive Model Approach to Two Dimensional Shape Classification", in IEEE Trans. on Pattern Analaysis and Machine Intelligence, Vol. PAM1-8, No. 1 pp. 55-66, Jan. 1986.

[11] B. Kartikeyan and A. Sarkar, "Shape Description by Time Series", in IEEE Trans. on Pattern Analaysis and Machine Intelligence, Vol. 11, No. 9 pp. 489-496, Sep. 1989. 


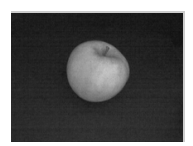

(a)

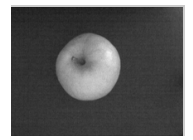

(g)

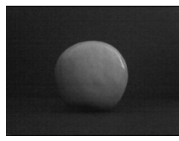

(m)

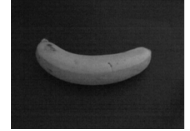

(b)

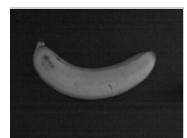

(h)

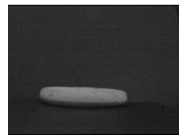

(n)

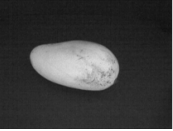

(c)

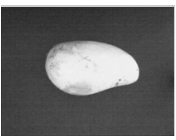

(i)

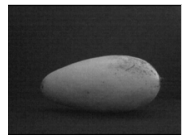

(o)

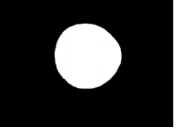

(d)

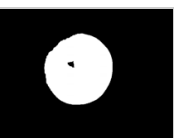

(j)

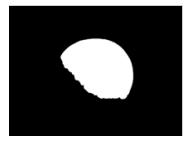

(p)

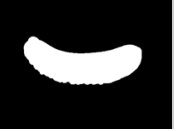

(e)

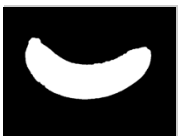

(k)

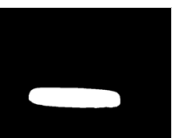

(q)

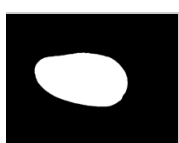

(f)

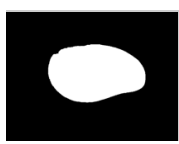

(1)

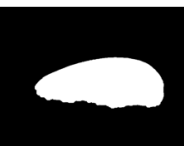

(r)

Figure 4. Fruit Images, (a-f) Fruits images acquired with the camera inclined at an angle of $60^{\circ}$; (g-l)Fruits images acquired with the camera inclined at top view position; (m-r)Fruits images acquired with the camera inclined at top view position

[12] A. F. Abtae, M. Nappi, D. Riccio and S. Ricciardi, " Ear Recognition by means of a Rotational Invariant Descriptor", Proc. of 18th Int. Conf. on Pattern Recognition, pp 437-440, 2006.

[13] A. El-Ghazal, O. Basir and S. Belkasim, "A New Shape Signature for Fourier Descriptors", 14th IEEE International Conference on Image Processing, San Antonio, Texas, USA, pp. 161-164, 2007.

[14] M. Zhenjiang, M.H Gandelin and Y. Baozong, "Fourier Based Image Shape Analysis and Its Application to Flower Recognition", IEEE 6th International Conference on Singal Processing, Ottawa, Canada, pp.1087-1090ICSP-2002.

[15] H. Jia and M. Xie, “ Improvement of Fourier Descriptor Using Spatial Normalization”, IEEE ISCIT-2005, pp. 1237$1240,2005$.

[16] H. Wu, P. Zhou and Z. Gao, "An Algorithm for Automatic Side Face Portrait Recognition based on Fourier Descriptor", IEEE Int. Symp. on Info. Sci and Engr, 2008, pp. 767-772, 2008.

[17] N. Tahir, A. Hussain, and M. Mustafa, " Fourier Descriptor for Pedestrian Shape Recognition usig Support Vector Machine ", IEEE Int. Symp. on Signal Proc. and Info. Tech., 2007, pp. 636-640, 2007.

[18] I. Kunttu and L. Lepstio, " Shape-based retrieval of Industrial Surface defects using angular radius Fourier Descriptor ", IET, 2007, pp. 231-236, 2007.

[19] Z. Wang, Z. Chi, and D. Feng, " Shape based leaf Image Retrieval ", IEE proc. Vis. Image Signal Process., Vol 150, No. 1 2003, pp. 34-43, 2003.
[20] M. H. Mahoor and M. Abdel-Mottaleb, " Automatic Classification of Teeth in BITEWING Dental Image", ICIP-2004, pp. 3475-3478, 2004.

[21] A.M. Aibinu, D. Kais, M.J.E. Salami, A.A. Shafie and A. Najeeb, "New Methods of Shape Identification Using Artificial Neural Network Based Autoregressive Modeling Techniques", submitted for publication in Machine Vision and Applications, 2010.

[22] MATLAB toolboox.

[23] N. Otsu, "A threshold selection method from gray-level histogram", IEEE Trans. Sys., Man., Cyber. 9: 62-66. doi:10.1109/TSMC.1 979.4310076

[24] S. Haykin, “ Neural Networks: A comprehensive foundation, 2nd ed.", Eaglewood, Cliffs, NJ: Prentice Hall.

[25] A. M. Aibinu, M. J. E. Salami, and A. A. Shafie, "Determination of Complex-Valued Parametric Model Coefficients Using Artificial Neural Network Technique", AANS Volume 2010 (2010), Article ID 984381.

[26] D.E. Rumelhart and J.L.MacClelland, Parallel Distributed Processing: Explorations in the Microstructure of Cognition, vol. I, MIT Press, Cambridge, MA, 1986. 